\title{
Methylated circulating tumor DNA as a biomarker in cutaneous melanoma
}

\author{
Russell J Diefenbach¹,2 (D), Jenny H Lee 1,2 iD \& Helen Rizos*,1,2 iD \\ ${ }^{1}$ Department of Biomedical Sciences, Faculty of Medicine, Health \& Human Sciences, Macquarie University, Sydney, NSW 2109, \\ Australia \\ ${ }^{2}$ Melanoma Institute Australia, The University of Sydney, Sydney, NSW 2065, Australia \\ *Author for correspondence: helen.rizos@mq.edu.au
}

"Exploring methylation patterns is a promising advancement in ctDNA analysis that will undoubtedly complement and enhance the highly sensitive approaches currently used for the detection of ctDNA mutations."

First draft submitted: 22 May 2020; Accepted for publication: 5 June 2020; Published online: 31 July 2020

Keywords: ctDNA • ddPCR • melanoma • methylation • next-generation sequencing

The detection and monitoring of circulating tumor DNA (ctDNA) in melanoma using mutation-detection techniques such as droplet digital PCR and targeted next-generation sequencing (NGS) panels has been well documented (reviewed in [1]). In melanoma, baseline ctDNA assessment predicts overall survival of stage III patients and longitudinal assessment predicts response and survival of stage IV patients treated with immune checkpoint inhibitors and targeted therapies [2-4]. ctDNA can also be used to monitor the appearance of treatment-resistant melanoma subclones [2], tumor heterogeneity [5], metabolic tumor burden [6] and differentiates true progression from pseudoprogression in melanoma patients treated with immunotherapy [7]. ctDNA can be detected in approximately 34\% of stage III melanoma and $73 \%$ of stage IV melanoma [2,4].

Several customized melanoma-associated NGS mutation panels for ctDNA have been described $[8,9]$, and these panels are designed to detect greater than $80 \%$ of melanomas with mutant allele frequency detection limits of only $0.1 \%$. These sequencing panels have several limitations, however. They often yield lower coverage of guaninecytosine (GC)-rich DNA regions, and these regions can be extremely informative cancer markers. For instance, the TERT gene has a GC-rich promoter that is mutated in approximately $70 \%$ of melanomas [8]. The complete sequencing of large genes (such as the $N F 1$ gene at $>8 \mathrm{~kb}$ ) is also difficult as the increased gene coverage comes at the expense of overall mutation detection sensitivity. Moreover, the significance of many, low-frequency mutations can be unclear and alterations in some genes, including the TP53 tumor suppressor gene, may not reflect tumor biology, but rather clonal hematopoiesis, a common age-associated phenomenon involving the expansion of nucleated blood cells with somatic mutations [10].

An alternative liquid biopsy approach for monitoring cancer, without prior knowledge of somatic mutation profiles, involves the detection of epigenetic DNA changes, such as methylation. Methylation of cytosine residues within $\mathrm{CpG}$ islands is important for the regulation of gene expression and is altered during the development and progression of many cancers, including melanoma. Importantly, aberrant methylation of CpG-rich gene promoters can be a very consistent feature of cancer [11] and thus, the analysis of methylated DNA in liquid biopsies is a rapidly emerging area of interest [12]. Patterns of DNA methylation can change during melanoma progression and the analysis of DNA methylation can provide valuable information related to the phenotypic behavior and stage of melanoma [13]. The analysis of ctDNA methylation is challenging due to the low amounts and highly fragmented nature of ctDNA. Typically, most methylation analysis workflows incorporate an initial bisulfite conversion step which preserves methylated cytosines prior to downstream sequencing. With limited amounts of ctDNA, it is imperative that the efficiency of bisulfite conversion nears 100\% [14], and we have recently achieved bisulfite conversion efficiency of $>99.5 \%$ when using $20 \mathrm{ng}$ of ctDNA [15].

Future Medicine 
There are several liquid biopsy based methylation assays for liver, lung, bladder and colorectal cancers which are based on monitoring DNA methylation using methylation specific PCR-based assays [16]. Targeted, predesigned tumor-specific methylation gene panels requiring bisulfite conversion and subsequent NGS of ctDNA, are also available for leukemia, lymphoma, prostate, breast and colorectal cancers from companies such as (Qiagen, Hilden, Germany) and (Thermofisher, MA, USA). The technologies for the analysis of methylated ctDNA are rapidly evolving and a thorough evaluation of these commercially available technologies and panels is now required to confirm their efficiency, specificity and sensitivity in a clinical setting.

In the case of melanoma, a predesigned methylation specific ctDNA panel, that includes markers of disease progression and response or resistance to therapies, is not yet available. There are ongoing efforts to optimize the selection of melanoma-specific methylation panels by exploring common hypermethylated and hypomethylated genetic changes in melanoma tissue $[13,17,18]$. The choice of gene targets is critical in order to achieve a high degree of sensitivity and specificity as there will be widespread age-specific and tissue-specific DNA methylation changes [19]. The inclusion of both hypermethylated and hypomethylated gene targets is also important. Hypermethylation can drive the silencing of tumor suppressor genes, while hypomethylation can lead to the activation of proto-oncogenes and their downstream signaling pathways. In the case of hypermethylation, the choice of gene promoter versus intragenic regions also needs to be considered, given that intragenic DNA methylation changes are more likely to be tissue-specific, and thus provide greater specificity for tumor detection [19]. Furthermore, methylation panels that assess the methylation of CpG sites on both DNA strands provide added value as there may be DNA strand differences in methylation across some gene targets [15]. Collectively, consideration of these variables will produce the most comprehensive melanoma-specific methylation ctDNA panel for monitoring melanoma.

Whether these methylation-specific panels can improve, match or add value to mutation-based ctDNA detection assays remains to be seen. Currently, ctDNA profiling detects less than $40 \%$ of high-risk, early stage melanoma [4] and is not an accurate marker of intracranial disease activity in patients with brain metastases [20]. The value of analyzing methylated ctDNA in these settings will be of particular importance. The additional clinical information gained by evaluating methylated ctDNA also needs thorough investigation - can methylated ctDNA profiles predict melanoma response to subsequent therapies, detect minimal residual disease after definitive surgery, detect melanoma recurrence during active surveillance and accurately stratify patients to inform the clinical management of high-risk, early stage and advanced melanoma patients? Moreover, can clinicians easily and accurately interpret the ctDNA mutation and methylation data to assist in the management of their patients? Methylation profiles will also evolve and change as we measure ctDNA during each patient's treatment journey and interpreting these changes will be essential if the goal is to manage patient care with personalized therapy in real time.

There has been significant progress in the analysis and application of liquid biopsies in cancer care, and there remain many opportunities for improvement - including improved specificity, sensitivity and expanded biomarker panels that reflect not only the presence of cancer, but the biology of the disease. Exploring methylation patterns is a promising advancement in ctDNA analysis that will undoubtedly complement and enhance the highly sensitive approaches currently used for the detection of ctDNA mutations.

\section{Author contributions}

All authors were responsible for drafting and revision of the manuscript.

Financial \& competing interests disclosure

RJ Diefenbach was supported in part by a donation to Melanoma Institute Australia from the Clearbridge Foundation. This work was also supported in part by the National Health and Medical Research Council (APP1093017 and APP1128951). JH Lee has received honorarium from AstraZeneca and travel support from Bristol Meyer Squibb. H Rizos is supported by a National Health and Medical Research Council, Research Fellowship. The authors have no other relevant affiliations or financial involvement with any organization or entity with a financial interest in or financial conflict with the subject matter or materials discussed in the manuscript apart from those disclosed.

No writing assistance was utilized in the production of this manuscript. 


\section{References}

1. Diefenbach RJ, Lee JH, Rizos H. Monitoring melanoma using circulating free DNA. Am. J. Clin. Dermatol. 20(1), 1-12 (2019).

2. Gray ES, Rizos H, Reid AL et al. Circulating tumor DNA to monitor treatment response and detect acquired resistance in patients with metastatic melanoma. Oncotarget 6(39), 42008-42018 (2015).

3. Lee JH, Long GV, Boyd S et al. Circulating tumour DNA predicts response to anti-PD1 antibodies in metastatic melanoma. Ann. Oncol. 28(5), 1130-1136 (2017).

4. Lee JH, Saw RP, Thompson JF et al. Pre-operative ctDNA predicts survival in high-risk stage III cutaneous melanoma patients. Ann. Oncol. 30(5), 815-822 (2019).

5. Diefenbach RJ, Lee JH, Strbenac D et al. Analysis of the whole-exome sequencing of tumor and circulating tumor DNA in metastatic melanoma. Cancers (Basel) 11(12), 1905 (2019).

6. Mcevoy AC, Warburton L, Al-Ogaili Z et al. Correlation between circulating tumour DNA and metabolic tumour burden in metastatic melanoma patients. BMC Cancer 18(1), 726 (2018).

7. Lee JH, Long GV, Menzies AM et al. Association between circulating tumor DNA and pseudoprogression in patients with metastatic melanoma treated with anti-programmed cell death 1 antibodies. JAMA Oncol. 4(5), 717-721 (2018).

8. Calapre L, Giardina T, Robinson C et al. Locus-specific concordance of genomic alterations between tissue and plasma circulating tumor DNA in metastatic melanoma. Mol. Oncol. 13(2), 171-184 (2019).

9. Gray ES, Witkowski T, Pereira M et al. Genomic analysis of circulating tumor DNA using a melanoma-specific UltraSEEK oncogene panel. J. Mol. Diagn. 21(3), 418-426 (2019).

10. Xie M, Lu C, Wang J et al. Age-related mutations associated with clonal hematopoietic expansion and malignancies. Nat. Med. 20(12), 1472-1478 (2014).

11. Moran B, Silva R, Perry AS, Gallagher WM. Epigenetics of malignant melanoma. Semin. Cancer Biol. 51, 80-88 (2018).

12. Constancio V, Nunes SP, Henrique R, Jeronimo C. DNA methylation-based testing in liquid biopsies as detection and prognostic biomarkers for the four major cancer types. Cells 9(3), 624 (2020).

13. Wouters J, Vizoso M, Martinez-Cardus A et al. Comprehensive DNA methylation study identifies novel progression-related and prognostic markers for cutaneous melanoma. BMC Med. 15(1), 101 (2017).

14. Worm Orntoft MB, Jensen SO, Hansen TB, Bramsen JB, Andersen CL. Comparative analysis of 12 different kits for bisulfite conversion of circulating cell-free DNA. Epigenetics 12(8), 626-636 (2017).

15. Diefenbach RJ, Lee JH, Chandler D et al. Hypermethylation of circulating free DNA in cutaneous melanoma. Appl. Sci. 9(23), 5074 (2019).

16. Locke WJ, Guanzon D, Ma C et al. DNA methylation cancer biomarkers: translation to the clinic. Front. Genet. 10, 1150 (2019)

17. Koroknai V, Szasz I, Hernandez-Vargas $\mathrm{H}$ et al. DNA hypermethylation is associated with invasive phenotype of malignant melanoma. Exp. Dermatol. 29(1), 39-50 (2020).

18. Jung H, Kim HS, Kim JY et al. DNA methylation loss promotes immune evasion of tumours with high mutation and copy number load. Nat. Commun. 10(1), 4278 (2019).

19. Ehrlich M. DNA hypermethylation in disease: mechanisms and clinical relevance. Epigenetics 14(12), 1141-1163 (2019).

20. Lee JH, Menzies AM, Carlino MS et al. Longitudinal monitoring of ctDNA in patients with melanoma and brain metastases treated with immune checkpoint inhibitors. Clin. Cancer Res. doi:10.1158/1078-0432.CCR-19-3926 (2020) (Epub ahead of print). 
